\title{
Asociación entre el alelo APOE- $\varepsilon 4$ en adultos mayores mexicanos con deterioro cognitivo
}

\author{
Israel de Jesús García-Muñoz
}

Secretaría de Salud, Instituto Nacional de Rehabilitación "Luis Guillermo Ibarra Ibarra", Servicio de Geriatría, Ciudad de México, México

El artículo "Programa de detección del alelo APOE- 44 en adultos mayores mexicanos con deterioro cognitivo", publicado en el número 5 de 2018 de Gaceta Médica de México" se presenta como el "primer estudio en México implementado para conocer el riesgo de ese alelo para trastornos neurocognitivos". Al respecto, es importante realizar algunas acotaciones:

- No es el primer estudio en población mexicana que analiza la presencia del alelo APOE- $\varepsilon 4$ en deterioro cognitivo. En 2008, Villalpando Berumen et al. ${ }^{2}$ publicaron una investigación que evaluó dicha asociación; no encontraron diferencias en las frecuencias de la presentación de los alelos APOE ع3 y $\varepsilon 4$ en sujetos con y sin criterios para demencia tipo Alzheimer ajustados por edad, sexo y escolaridad a diferencia de los resultados señalados por Genis Mendoza et al.
- El alelo APOE- 4 se ha establecido como un factor de riesgo para demencia tipo Alzheimer familiar y esporádica, pero no se ha encontrado asociación significativa para otros tipos de demencia o trastornos neurocognitivos. Considerando lo anterior, en el artículo que aquí comentamos no se categoriza ni se estratifica a la población estudiada con trastorno neurocognitivo mayor con diagnósticos de probabilidad para los diferentes tipos de demencia y así establecer una asociación más sólida y fidedigna.

\section{Bibliografía}

1. Genis-Mendoza AD, Martínez-Magaña JJ, Bojórquez C, Téllez-Martínez JA, Jiménez-Genchi J, Roche A, et al. Programa de detección del alelo APOE- $\varepsilon 4$ en adultos mayores mexicanos con deterioro cognitivo. Gac Med Mex. 2018;154:555-560.

2. Villalpando-Berumen JM, Mejía-Arango S, Aguilar-Salinas CA, Ordónez-Sánchez ML, Gutiérrez-Robledo LM. Apolipoprotein E epsilon4, Alzheimer's disease, and cognitive performance in elderly Mexican mestizos. J Am Geriatr Soc. 2008;56:677-682.
Gac Med Mex. 2019;155:110-110 Disponible en PubMed www.gacetamedicademexico.com 